\section{Is the Regulation of Microtu- bule Stability at the Crossroad between Aging and Disease of Dopaminergic neurons?}

\author{
Cartelli Daniele ${ }^{\star 1}$ and Cappelletti Graziella ${ }^{1,2}$ \\ ${ }^{1}$ Department of Biosciences, Università degli Studi di Milano, Milano, Italy \\ ${ }^{2}$ Center of Excellenceon Neurodegenerative Diseases, Università degli \\ Studi di Milano, Milano, Italy
}

\begin{abstract}
Over the time, it is becoming clear that microtubule system plays a fundamental role not only during the development of central nervous system but also during brain aging and neuronal death. In particular, the fine modulation of microtubule stability and tubulin posttranslational modifications is crucial for neurons as its failure induces brain damage. Basing our idea on the facts that dopaminergic neurons possess a peculiar architecture, strictly dependant on microtubule integrity, and that most of the experimental models of Parkinson's disease undergo microtubule destabilization before dopaminergic cells loss, here we propose that the regulation of microtubule stability can be the Achilles' heel of this neuronal population. Therefore, we hypothesize that failure of the modulation of microtubule system could represent the crossroad between normal brain aging and neurodegenerative processes.
\end{abstract}

Keywords: Dopaminergic neuron; Microtubule stability; Parkinson's disease; Tubulin acetylation

Microtubules (MTs) are built up by $\alpha \beta$ tubulin dimers, which assemble into non-covalent polymers with an intrinsic resistance to bending and compression and capable to alternate slow polymerizing phases to rapid shrinking ones, a behaviour called "dynamic instability" [1]. The proper regulation of MT stability (i.e., more stable MTs display a longer half-life) is fundamental for the development, the survival and the aging of neurons, since both hyper-stable and overly dynamic MTs affect neuronal functions eventually leading to cell death [2]. $\alpha$ and $\beta$ tubulin Posttranslational Modifications (PTMs) mark dynamic or stable MTs, which are found in specific neuronal compartments and whose stability changes during neuronal differentiation and aging [3]. Indeed, tyrosinated $\alpha$ tubulin is associated to highly dynamic MTs, which are abundant in young neurons and at the

*Corresponding author: Daniele Cartelli, Department of Biosciences, Università degli Studi di Milano, Via Celoria 26, Milano, 20133, Italy, Tel: +39 3334920793; E-mail: daniele.cartelli@gmail.com

Citation: Cartelli D, Cappelletti G (2017) Is the Regulation of Microtubule Stability at the Crossroad between Aging and Disease of Dopaminergic neurons? J Alzheimers Neurodegener 3: 010.

Received: November 22, 2016; Accepted: January 24, 2017; Published: February 07, 2017 presynaptic bouton of mature neurons. During neuronal maturation MT stability increases and MTs inside the axonal shaft accumulate PTMs which extend their half-life, either through a direct stabilization of the MT lattice [4] or by governing upstream events, such as the recruitment of MT-interacting protein [5] or by protecting MTs from depolymerizing kinesins and severing enzymes [6,7]. Nevertheless, the stability of MTs has to be maintained under a physiological threshold, since their excessive stabilization can eventually lead to MT bundling and block of axonal transport [8]. As many of the axonal MTs are long-lived polymers which persist for the entire life of the neuron, their aging is a critical event which potentially impacts on the neuron lifespan. Indeed, MTs are structures with an intrinsic self-repair capability [9] but, due to defects accumulated during growth [10] and to their ends which become more tapered during time [11], the older MTs are more susceptible to catastrophes than younger ones [12,13]. Interestingly, tubulin PTMs are potential regulators of this process as much as, for long time, acetylated MTs were referred almost synonymously to old and stable MTs. Indeed, among its several biological functions [14], it has been shown that acetylation accumulates during MT aging and it has been proposed that the MT-acetylase $\alpha$ Tubulin Acetyl Transferase1 (aTAT1) can act as a clock for MT lifetimes [15], due to its preference for MTs and its low catalytic rate. Nevertheless, other candidates may be responsible for minor tubulin acetylation in vivo, including Elongator Complex Protein Elp3, which acts as tubulin acetylase, controlling the development and the migration of cortical neurons [16]. Furthermore, the age-dependent decrease of the activity of sirtuin 1, a widespread deacetylase which also acts on MTs, could lead to increased acetylation of a tubulin in the cerebellum of aged mice [17]. These data suggest a functional link between MT acetylation patterns and brain aging, evidencing how the regulation of MT stability is critical during normal neuronal aging and supporting the concept that its failure may be a reliable candidate in causing neurodegenerative processes. Indeed, during the last years, an increasing body of evidence indicated the potential role of defective regulation of MT stability in neurodegenerative disorders [18] and, in particular, in Parkinson's Disease (PD) [19,20].

$\mathrm{PD}$ is the second most common neurodegenerative disorder caused by the death of the dopaminergic neurons in the Substantia nigra [21] whose anatomy, with an average arborisation of 4.6 meters in humans [22], makes them particularly dependant on axonal transport which, in turn, depends on MT integrity and whose failure has been associated to the early phases of PD [23]. Although the identity of the real culprit of the pathology is still debated, in the recent years it is becoming ever clearer that MT destabilization is an early event in experimental and human models of $\mathrm{PD}$, and many of the studies reported below strongly suggest that it can be followed by the hyper-stabilization of MTs, which could lead to detrimental side effects. Indeed, during the last decade, we have demonstrated that $\mathrm{MPP}^{+}$, the metabolite of the PD-inducing toxin MPTP, acts as a catastrophe promoter, leading to the earlier MT destabilization and to a later excessive accumulation of tubulin PTMs associated to stable MTs (detyrosination and acetylation), in vitro as well as in vivo [24-26]. Similarly, 6-hydroxydopamine elicits significant alterations in MT dynamics, 
including reduction in MT growth rate and overload of a tubulin acetylation [27]. Furthermore, MT fragmentation has been observed in various dopaminergic neuronal models exposed to various PD-inducing neurotoxin [28-30 and Casagrande et al., personal communication], and Ren and colleagues clearly demonstrated that dopaminergic neurons are much more susceptible to MT destabilization than other neuronal types [28]. Moreover, some and perhaps the most important PD-related proteins interact with the MT system modulating its assembly and stability. We recently demonstrated that wild type a-Synuclein is a MT dynamase which regulates MT nucleation and dynamics and whose PD-associated mutations induce tubulin aggregation [31]. Both parkin and Leucine Rich Repeat Kinase 2 (LRRK2) interact with and stabilize MTs [32,33] and, notably, induced Pluripotent Stem Cells (iPSC)-derived neurons obtained from patients carrying mutations in parkin and LRRK2 display MT-destabilization or tubulin aggregation [34,35]. It is intriguing that the actions of parkin and LRRK2 converge on MTs acetylation and axonal transport, two molecular events that are strictly correlated, since tubulin acetylation speeds up anterograde transport of synaptic vesicles [36] and compensates for the transport defects observed in Huntington's disease [37]. PD-associated Roc-COR domain LRRK2 mutants preferentially bind to deacetylated MTs inhibiting axonal transport in primary neurons of Drosophila melanogaster and causing locomotor deficits; interestingly, both the genetic and pharmacological increasing of MT acetylation is able to rescue these defects in vivo [38]. Our unpublished results suggest that parkin knockout mouse shows an accelerated accumulation of acetylated MTs, which is specifically associated to dopaminergic neurons, and which can easily lead to the block of mitochondrial transport we observed [Cartelli et al., unpublished]. We have now to remember that both neuronal health [2] and axonal transport [36,37] are sensitive to either overly dynamic (deacetylated) or hyper-stable (heavily acetylated) MTs, as the ones that overcame the lower or the upper MT stability-limiting physiological threshold. Therefore, all these results reinforce the idea that the fine tuning of MT stability, and in particular the modulation of MT acetylation inside dopaminergic neurons, is a critical event during brain aging, and suggest that its failure can be the crossroad between normal neuronal aging and neurodegenerative processes.

The above described MT-based neuronal defects can be counteracted by the administration of MT-stabilizing agents, as already suggested $[19,39]$ and demonstrated in many of the studies above reported $[26,28,34]$. A reliable alternative can be the fine modulation of MT acetylation, which seems to be tightly associated to axonal transport and locomotor defects $[16,36,38]$. Since the extent of MT acetylation depends on the balance of the antagonistic acetylases and deacetylases activities, its modulation can be achieved by the use of specific stimulators or inhibitors of aTAT1 or Histone Deacetylase 6 (HDAC6) [14]. The latter specifically acts on tubulin [40], and its inhibition exerts neuroprotection in many age-related neurodegenerative disorders [41] and rescues the transport defects observed in Huntington's disease [37]. In agreement, good results have been achieved in PD experimental models by using inhibitors of MT-deacetylases, as reported by Godena et al., [38] or by Outiero and colleagues [42], which can counteract the excessive accumulation of acetylated MTs or, in other words, the unusual MT hyper-stabilization. Since acetylation marks old MTs and older MTs are more prone to depolymerise that younger ones [12], a further possibility could be trying to induce a sort of MT rejuvenation. This aim does not strictly require the inhibition of MT acetylation but, in order to reach this goal, one could simply stimulate tubulin PTMs associated to newly synthetized and highly dynamic
MTs, as a tubulin tyrosination, which should have beneficial effects also on the neuron itself. Indeed, the specific and unique Tubulin Tyrosine Ligase (TTL) enzyme increases the levels of tyrosinated $\alpha$ tubulin at the axonal injury site, activating pro-regenerative transcriptional program important for axon regrowth [43].

Putting all the pieces together, here we propose that the regulation of MT stability, and in particular the modulation of MT acetylation, can be the Achilles' heel of the dopaminergic system, as its weakness could actually discriminate between aging and degeneration. A remaining question is how the disruption of such a ubiquitous process can affect selected neuronal populations and induce specific neurological phenotypes. Our personal feeling is that the answer has to be looked for in the anatomy of neuronal subtypes; indeed, dopaminergic neurons have very long axons whose maintenance and function depend on the correct executions of axonal transport processes and, thus, on MT health, whatever it does mean. In agreement, other long-distance projecting neuronal populations, as striatal and motor neurons, do show alterations of MT stability regulation in pathological contexts $[37,44,45]$. Therefore, we firmly believe that the failure of a crucial and fine regulated process during brain aging, like the control of MT stability is supposed to be, can specifically affect those neuronal subpopulations whose requirements for MT integrity is pressing and, therefore, it could induce pathological states.

\section{Acknowledgement}

This work was supported by Fondazione Grigioni per Il Morbo Di Parkinson, Milan, Italy [to G.C.]; "Dote Ricerca”, FSE, Regione Lombardia [to D.C.]. The authors are grateful to all the members of the lab and to all the people which contribute in delineating this story. The authors declare that they have no conflict of interest.

\section{References}

1. Mitchison T, Kirschner M (1984) Dynamic instability of microtubule growth Nature 312: 237-242.

2. Feinstein $S C$, Wilson $L$ (2005) Inability of tau to properly regulate neuronal microtubule dynamics: a loss-of-function mechanism by which tau might mediate neuronal cell death. Biochim Biophys Acta 1739: 268-279.

3. Janke C, Kneussel M (2010) Tubulin post-translational modifications: encoding functions on the neuronal microtubule cytoskeleton. Trends Neurosci 33: 362-372.

4. Song Y, Kirkpatrick LL, Schilling AB, Helseth DL, Chabot N, et al. (2013) Transglutaminase and polyamination of tubulin: posttranslational modification for stabilizing axonal microtubules. Neuron 78: 109-123.

5. Bonnet C, Boucher D, Lazereg S, Pedrotti B, Islam K, et al. (2001) Differential binding regulation of microtubule-associated proteins MAP1A, MAP1B, and MAP2 by tubulin polyglutamylation. J Biol Chem 276: 12839-12848.

6. Peris L, Wagenbach M, Lafanechère L, Brocard J, Moore AT, et al. (2009) Motor-dependent microtubule disassembly driven by tubulin tyrosination. $J$ Cell Biol 185: 1159-1166.

7. Sharp DJ, Ross JL (2012) Microtubule-severing enzymes at the cutting edge J Cell Sci 125: 2561-2569.

8. Salinas S, Carazo-Salas RE, Proukakis C, Schiavo G, Warner TT (2007) Spastin and microtubules: Functions in health and disease. J Neurosci Res 85: $2778-2782$

9. Schaedel L, John K, Gaillard J, Nachury MV, Blanchoin L, et al. (2015) Microtubules self-repair in response to mechanical stress. Nat Mater 14: 11561163.

10. Bowne-Anderson H, Zanic M, Kauer M, Howard J (2013) Microtubule dynamic instability: a new model with coupled GTP hydrolysis and multistep catastrophe. Bioessays 35: 452-461. 
Citation: Cartelli D, Cappelletti G (2017) Is the Regulation of Microtubule Stability at the Crossroad between Aging and Disease of Dopaminergic neurons? J Alzheimers Neurodegener 3: 010.

11. Coombes CE, Yamamoto A, Kenzie MR, Odde DJ, Gardner MK (2013) Evolving tip structures can explain age-dependent microtubule catastrophe. Curr Biol 23: 1342-1348.

12. Gardner MK, Zanic M, Gell C, Bormuth V, Howard J (2011) Depolymerizing kinesins Kip3 and MCAK shape cellular microtubule architecture by differential control of catastrophe. Cell 147: 1092-1103.

13. Duellberg C, Cade NI, Surrey T (2016) Microtubule aging probed by microfluidics-assisted tubulin washout. Mol Biol Cell 27: 3563-3573.

14. Li L, Yang XJ (2015) Tubulin acetylation: responsible enzymes, biological functions and human disease. Cell Mol Life Sciences 72: 4237-4255.

15. Szyk A, Deaconescu AM, Spector J, Goodman B, Valenstein ML, et al. (2014) Molecular basis for age-dependent microtubule acetylation by tubulin acetyltransferase. Cell 157: 1405-1415.

16. Creppe C, Malinouskaya L, Volvert ML, Gillard M, Close P, et al. (2009) Elongator controls the migration and differentiation of cortical neurons through acetylation of alpha-tubulin. Cell 136: 551-564

17. Marton O, Koltai E, Nyakas C, Bakonyi T, Zenteno-Savin T, et al. (2010) Aging and exercise affect the level of protein acetylation and SIRT1 activity in cerebellum of male rats. Biogerontology 11: 679-686.

18. Baas PW, Rao AN, Matamoros AJ, Leo L (2016) Stability properties of neuronal microtubules. Cytoskeleton (Hoboken) 73: 442-460.

19. Cartelli D, Cappelletti G, (2016) Microtubule Destabilization Paves the Way to Parkinson's Disease. Mol Neurobiol 1-13.

20. Pellegrini L, Wetzel A, Grannó S, Heaton G, Harvey K (2017) Back to the tubule: microtubule dynamics in Parkinson's disease. Cell Mol Life Sci 74 409-434.

21. Obeso JA, Rodriguez-Oroz MC, Goetz CG, Marin C, Kordower JH, et al (2010) Missing pieces in the Parkinson's disease puzzle. Nat Med 16: 653661

22. Pissadaki EK, Bolam JP (2013) The energy cost of action potential propagation in dopamine neurons: clues to susceptibility in Parkinson's disease. Fron Comput Neurosci 7: 13.

23. Hunn BH, Cragg SJ, Bolam JP, Spillantini MG, Wade-Martins R (2015) Impaired intracellular trafficking defines early Parkinson's disease. Trends Neurosci 38: 178-88.

24. Cappelletti G, Surrey T, Maci R (2005) The parkinsonism producing neurotoxin MPP ${ }^{+}$affects microtubule dynamics by acting as a destabilising factor. FEBS Lett 579: 4781-4786

25. Cartelli D, Ronchi C, Maggioni MG, Rodighiero S, Giavini E, et al. (2010) Microtubule dysfunction precedes transport impairment and mitochondria damage in MPP+-induced neurodegeneration. J Neurochem 115: 247-258.

26. Cartelli D, Casagrande F, Busceti CL, Bucci D, Molinaro G, et al. (2013) Microtubule alterations occur early in experimental parkinsonism and the microtubule stabilizer epothilone D is neuroprotective. Sci Rep 3: 1837.

27. Patel VP, Chu CT (2014) Decreased SIRT2 activity leads to altered microtubule dynamics in oxidatively-stressed neuronal cells: implications for Parkinson's disease. Exp Neurol 257: 170-181.

28. Ren Y, Liu W, Jiang H, Jiang Q, Feng J (2005) Selective vulnerability of dopaminergic neurons to microtubule depolymerization. J Biol Chem 280: 3410534112.
29. Escobar-Khondiker M, Höllerhage M, Muriel MP, Champy P, Bach A, et al. (2007) Annonacin, a natural mitochondrial complex I inhibitor, causes tau pathology in cultured neurons. J Neurosci 27: 7827-7837.

30. Kim-Han JS, Antenor-Dorsey JA, O'Malley KL (2011) The parkinsonian mimetic, MPP ${ }^{+}$, specifically impairs mitochondrial transport in dopamine axons. J Neurosci 31: 7212-7221

31. Cartelli D, Aliverti A, Barbiroli A, Santambrogio C, Ragg EM, et al. (2016) a-Synuclein is a Novel Microtubule Dynamase. Sci Rep 6: 33289.

32. Yang F, Jiang Q, Zhao J, Ren Y, Sutton MD, et al. (2005) Parkin stabilizes microtubules through strong binding mediated by three independent domains. J Biol Chem 280: 17154-17162.

33. Gillardon F (2009) Leucine-rich repeat kinase 2 phosphorylates brain tubulin-beta isoforms and modulates microtubule stability--a point of convergence in parkinsonian neurodegeneration? J Neurochem 110: 1514-1522.

34. Ren Y, Jiang H, Hu Z, Fan K, Wang J, et al. (2015) Parkin mutations reduce the complexity of neuronal processes in iPSC-derived human neurons. Stem Cells 33: $68-78$

35. Schwab AJ, Ebert AD (2015) Neurite Aggregation and Calcium Dysfunction in iPSC-Derived Sensory Neurons with Parkinson's Disease-Related LRRK2 G2019S Mutation. Stem Cell Reports 5: 1039-1052.

36. Reed NA, Cai D, Blasius TL, Jih GT, Meyhofer E, et al. (2006) Microtubule acetylation promotes kinesin-1 binding and transport. Curr Biol 16: 2166 2172.

37. Dompierre JP, Godin JD, Charrin BC, Cordelieres FP, King SJ, et al. (2007) Histone deacetylase 6 inhibition compensates for the transport defecit in Huntington's disease by increasing tubulin acetylation. J Neurosci 27: 3571-3583.

38. Godena VK, Brookes-Hocking N, Moller A, Shaw G, Oswald M, et al. (2014) Increasing microtubule acetylation rescues axonal transport and locomoto deficits caused by LRRK2 Roc-COR domain mutations. Nat Commun 5 : 5245.

39. Baas PW, Ahmad FJ (2013) Beyond taxol: microtubule-based treatment of disease and injury of the nervous system. Brain 136: 2937-2951.

40. Hubbert C, Guardiola A, Shao R, Kawaguchi Y, Ito A, et al. (2002) HDAC6 is a microtubule-associated deacetylase. Nature 417: 455-458.

41. Simões-Pires C, Zwick V, Nurisso A, Schenker E, Carrupt PA, et al. (2013) HDAC6 as a target for neurodegenerative diseases: what makes it different from the other HDACs? Mol Neurodegener 8: 7

42. Outeiro TF, Kontopoulos E, Altmann SM, Kufareva I, Strathearn KE, et al. (2007) Sirtuin 2 inhibitors rescue alpha-synuclein-mediated toxicity in models of Parkinson's disease. Science 317: 516-519.

43. Song W, Cho Y, Watt D, Cavalli V (2015) Tubulin-Tyrosine Ligase (TTL)-mediated Increase in Tyrosinated a-Tubulin in Injured Axons Is Required for Retrograde Injury Signaling and Axon Regeneration. J Biol Chem 290: 1476514775.

44. Evans KJ, Gomes ER, Reisenweber SM, Gundersen GG, Lauring BP (2005) Linking axonal degeneration to microtubule remodelling by Spastin-mediated microtubule severing. J Cell Biol 168: 599-606.

45. Piermarini E, Cartelli D, Pastore A, Tozzi G, Compagnucci C, et al. (2016) Frataxin silencing alters microtubule stability in motor neurons: Implications for Friedreich's ataxia. Hum Mol Genet. 